\title{
Potential Impact of Air Pollution on Multiple Sclerosis in Tehran, Iran
}

\author{
Pouria Heydarpour ${ }^{\mathrm{a}}$ Hassan Amini ${ }^{\mathrm{b}-\mathrm{d}}$ Shayan Khoshkish ${ }^{\mathrm{a}}$ Hossein Seidkhani ${ }^{\mathrm{e}}$ \\ Mohammad Ali Sahraian $^{a}$ Masud Yunesian $^{f}$ \\ ${ }^{a}$ MS Research Center, Neuroscience Institute, Tehran University of Medical Sciences, Tehran, Iran; ${ }^{b}$ Department of \\ Epidemiology and Public Health, Swiss Tropical and Public Health Institute, ' University of Basel, Basel, Switzerland; \\ ${ }^{\mathrm{d}}$ Kurdistan Environmental Health Research Center, Kurdistan University of Medical Sciences, Sanandaj, e Lab of Systems \\ Biology and Bioinformatics, Institute of Biochemistry and Biophysics, University of Tehran, and ${ }^{f}$ Center for Air Pollution \\ Research (CAPR), Institute for Environmental Research (IER), Tehran University of Medical Sciences, Tehran, Iran
}

\section{Key Words}

Air pollution · Iran · Multiple sclerosis · Particulate matter trols. Conclusion: This study revealed the potential role of long-term exposure to air pollutants as an environmental risk factor in MS.

(c) 2014 S. Karger AG, Basel

\begin{abstract}
Background: Multiple sclerosis (MS) incidence has dramatically increased in Tehran, Iran. The health impact of air pollution in Tehran underscores the attention to a possible association to this environmental risk factor. In this study, the authors aimed to analyze the spatial distribution of prevalent MS cases and their association with the spatial patterns of air pollution. Methods: Patient records meeting McDonald's criteria for definite MS diagnosis with disease onset during 2003-2013 were obtained. Next, the location of 2,188 patients was successfully geo-referenced within Tehran metropolis by geographic information system (GIS) bureau of Iran's post office based on their phone numbers. A cluster analysis was performed using the average nearest neighbor index (ANNI) and quadrat analysis. The long-term exposures of MS patients to particulate matter $\left(\mathrm{PM}_{10}\right)$, sulfur dioxide $\left(\mathrm{SO}_{2}\right)$, nitrogen oxide ( $\left.\mathrm{NO}\right)$, nitrogen dioxide $\left(\mathrm{NO}_{2}\right)$, and nitrogen oxides ( $\mathrm{NOx}$ ) were estimated using the previously developed land use regression models. Results: Prevalent MS cases had a clustered pattern in Tehran. A significant difference in exposure to $\mathrm{PM}_{10}, \mathrm{SO}_{2}, \mathrm{NO}_{2}$, and $\mathrm{NOx}$ $(p<0.001)$ was observed in MS cases compared with con-
\end{abstract}

\section{Introduction}

Multiple sclerosis (MS) is an inflammatory demyelinating disease affecting more than two million people worldwide [1]. Meta-regression analyses of studies on MS epidemiology has revealed an almost universal increase in prevalence and incidence of MS over time, and a general increase in incidence of MS in females has been observed [2]. Several studies suggest that MS incidence and prevalence have dramatically increased in Tehran over the last two decades [3-5]. Findings from the Global Burden of Disease (GBD) 2010 study suggested that disability-adjusted life years (DALYs) lost due to MS increased from 1990 to 2010 for both genders and all age groups in Iran. Ranks of death and DALYs attributable to MS in Iranian women increased from rank 90 to 74 and 122 to 105 , respectively in this period [6-8].

Pouria Heydarpour and Hassan Amini are equal first author contributors.

\section{KARGER}

E-Mail karger@karger.com

www.karger.com/ned
(C) 2014 S. Karger AG, Basel

0251-5350/14/0434-0233\$39.50/0
Prof. Masud Yunesian

Center for Air Pollution Research (CAPR)

Institute for Environmental Research (IER)

Tehran University of Medical Sciences, Tehran (Iran)

E-Mail yunesian@tums.ac.ir 
Sunlight exposure has been hypothesized to be a likely environmental candidate to explain the geographic variations in MS prevalence and the change in risk among migrants [9]. To date, the role of vitamin D in MS pathogenesis is the most biologically plausible explanation for a disproportional increase of MS among women $[2,10]$. Studies in Iran and India comparing areas with different grades of air pollution observed higher prevalence of vitamin $\mathrm{D}$ deficiency in women and toddlers in the more polluted sites $[11,12]$. However, knowledge of the association between the spatial distribution of air pollution and the prevalence of MS in Iran is currently lacking.

Today, Tehran is well-known for high levels of air pollution. Previous studies reported that this megacity is heavily polluted and the entire population is exposed to air pollutants well above the air quality guideline values of the World Health Organization [13, 14]. Spatial distribution of prevalent MS cases in Georgia, United States, suggested that urban attributes, especially particulate matter with aerodynamic diameter $\leq 10 \mu \mathrm{m}\left(\mathrm{PM}_{10}\right)$ may have a potential role in the etiology of MS in females [15]. In addition, there are studies suggesting that air pollutants $\left(\mathrm{PM}_{10}\right)$ could enhance the susceptibility of MS patients to infections and increase the relapse rate among them $[16,17]$. In this study, the authors aimed to analyze the spatial distribution of MS incident cases to investigate its association with long-term exposure to air pollution. The study is based on integrating health system and environmental data from various sources.

\section{Methods}

\section{Study Area}

This population-based study was conducted in Tehran, Iran (Latitude: $35^{\circ}$ North, Longitude: $51^{\circ}$ East). The weather is typically sunny, with average annual bright sunshine of $2,800 \mathrm{~h}$ and an annual mean cloud cover of $30 \%$. The average elevation is approximately 1,200 $\mathrm{m}$ above sea level, and the city covers a large area of $613 \mathrm{~km}^{2}$. Tehran has an urban resident population of over $8.2 \mathrm{mil}-$ lion people, though the daytime population has been estimated at more than 10 million people due to diurnal migration from the outlying areas $[13,18]$.

\section{Prevalent MS Cases}

Iranian MS Society (IMSS) records were studied to obtain annual incidence data. Briefly, the IMSS is the only center in the surveyed area that registers MS patients and provides wide facilities (medical, rehabilitation, and vast social welfare) for the members. Only patients approved by neurologists with MS fulfilling McDonald or the Poser criteria (up to 2001) are registered in the IMSS. A trained interviewer explains the purpose of the registry for patients in the IMSS, and after obtaining informed consent, patients are asked to complete structured interview questions [4]. Records of 2,188 patients meeting McDonald's criteria for definite MS diagnosis were obtained from IMSS (with disease onset during 20032013). Thereafter, they were successfully geo-referenced within Tehran by GIS bureau of Iran's post office based on their provided residence phone number.

\section{Cluster Analysis}

We applied two methods, namely average nearest neighbor index (ANNI) and quadrat analyses within a GIS to measure the clustering pattern of MS patients from 2003 to 2013 [19-21]. In the ANNI analysis, we calculated the distance between each MS case and the nearest MS case. Thereafter, we calculated the average distance between MS cases and compared with the distance of random cases within the study area. If the observed average distance of the MS cases was smaller than expected average distance of random distribution (nearest neighbor ratio), we considered the MS patients to have a clustered pattern $[22,23]$.

In quadrat analysis, we have overlayed the areas of equal size on the study area - known as quadrats or quads - and counted the number of MS cases in each square quadrat. One of the main points in this analysis is the size of the applied quads. Traditionally, the size of quads is identified as twice the size of the mean area per feature [24]. Thus, we have applied the following equation to calculate the length of a side of each quadrat:

$$
L=\sqrt{\left\{2 \times\left(\frac{A}{N}\right)\right\}} .
$$

where $L$ is the length of a side of each quadrat, $A$ is the area of extend for MS cases, and $N$ denoted the total observed number of MS cases. Thereafter, we have calculated the expected counts of MS cases for a random distribution in the study area based on Poisson distribution. We first calculated the probability of $x$ number of MS cases occurring in any given quadrat or $P(x)$ as the following equation:

$$
P(x)=\frac{e^{-\lambda} \lambda^{X}}{X !} .
$$

where $e$ is the Euler's constant, $X$ is the observed number of MS cases, and $\lambda$ is the average number of MS cases per quad. In addition, $\lambda$ was calculated based on the following equation:

$$
\lambda=\frac{n}{k} .
$$

where $n$ is the total number of MS cases and $k$ is the total number of quads. We then multiplied the $P(x)$ results by the total number of MS cases to get the number of quads expected to contain that number of MS cases. Therefore, we created two frequency tables: one for observed distribution, observed proportion, and observed cumulative proportion and the other one for expected distribution, expected proportion, and expected cumulative proportion based on Poisson distribution. We compared the largest absolute difference of the observed and expected cumulative proportions with the critical value of Kolmogorov-Smirnov test [21]. If the largest absolute difference was greater than the critical value, we considered the difference statistically significant and rejected the null hypothesis that MS cases have random distribution over the study area. 
Long-Term Air Pollution Exposure Assessment

We have estimated long-term exposure of all MS cases using previously developed land use regression (LUR) models for 2010 calendar year to estimate long-term exposure to air pollutants in Tehran $[13,18,25]$. In brief, hourly data for particulate matter $\left(\mathrm{PM}_{10}\right)$, sulfur dioxide $\left(\mathrm{SO}_{2}\right)$, nitrogen oxide $(\mathrm{NO})$, nitrogen dioxide $\left(\mathrm{NO}_{2}\right)$, and nitrogen oxides (NOx) were obtained from 23 air quality monitoring stations in 2010 . We then generated 210 potentially predictive variables in six classes and seventy-three sub-classes within GIS and used as spatial predictors. The six classes were traffic surrogates, land use, distance variables, population density, product variables, and geographic location. Multiple linear regression was used to correlate the measured air pollutant concentrations with the most predictive variables. A standard approach was developed for the LUR model building and finally the resulting equation was used to estimate the outdoor pollutant concentrations at the residential address for each individual MS patient within the city. These estimates were assumed to reliably reflect the spatial distribution of pollution during the entire seven-year period as seen elsewhere [26]. We have also created 8,752 random controls (fourfold of MS cases) as the control group using Geospatial Modeling Environment software, version 0.7.2.1 RC2 (GUI) (Spatial Ecology, LLC), and estimated individual exposure of each control. The independent samples t test was used to compare the difference in exposure to various air pollutants in MS patients and random points.

\section{Results}

\section{Cluster Analysis}

The ANNI analysis revealed that there was a clustered pattern for the MS cases in 2003-2013 in Tehran. There was less than $1 \%$ likelihood that this clustered pattern could be the result of random chance (nearest neighbor ratio $=0.56 ; \mathrm{Z}$ score $=-39.4$ standard deviations; $\mathrm{p}<$ $0.001)$. The length of the quads was calculated as 768 meters. The largest absolute difference between observed and expected cumulative proportions in the frequency table was 0.35 , while the critical value of KolmogorovSmirnov test was 0.03 , and this again demonstrates that MS cases have a clustered pattern in Tehran.

\section{MS and Air Pollution}

As depicted in figure 1, various air pollutant levels were estimated based on LUR models and MS patients were located on the map and their exposure to air pollutants was determined. Our analysis demonstrated that the annual mean exposure of $\mathrm{PM}_{10}, \mathrm{SO}_{2}, \mathrm{NO}, \mathrm{NO}_{2}$, and $\mathrm{NOx}$ in MS cases were $99.1 \mu \mathrm{g} / \mathrm{m}^{3}, 58$ parts per billion (ppb), 125.2, 48.1, and $113 \mathrm{ppb}$, respectively. However, in our random controls, the mean exposures for the mentioned pollutants were $93 \mu \mathrm{g} / \mathrm{m}^{3}, 52.5 \mathrm{ppb}, 108.9,41.1$, and 102 ppb, respectively.

Potential Impact of Air Pollution on Multiple Sclerosis
Various air pollutants had an exponential distribution and were significantly inter-correlated $(\mathrm{p}<0.001)$ except for $\mathrm{NO}$ and $\mathrm{SO}_{2}$. Principal component analysis revealed that the main effect of air pollutants could be attributed to $\mathrm{PM}_{10}$ and $\mathrm{SO}_{2}$.

A significant difference ( $\mathrm{p}<0.001$, independent samples $t$ test) in exposure to $\mathrm{PM}_{10}, \mathrm{SO}_{2}, \mathrm{NO}_{2}$, and $\mathrm{NOx}$ but not for NO was observed in MS cases compared with random controls generated on the Tehran map as the proxy control group.

\section{Discussion}

In this study, we first tried to analyze the pattern of MS incident cases to find out whether there is a clustered pattern in Tehran. We then tried to investigate the relationship of exposure to long-term air pollution in MS cases with the exposure of a control group as a proxy for exposure of general population in Tehran. In the absence of control of any potential confounding variables, the analyses are restricted to a comparison of those spatial patterns. Our results indicated that (a) the MS cases in Tehran had a clustered pattern, and (b) there was a statistically significant spatial correlation between the clustering of MS cases and the concentration patterns of $\mathrm{PM}_{10}, \mathrm{SO}_{2}$, $\mathrm{NO}_{2}$, and $\mathrm{NOx}$.

A recent study in Tehran demonstrated that a higher risk of MS in the northern zones of this mega city had a direct relation to the socioeconomic status of their inhabitants [27]. A clustered pattern of MS prevalence was also observed in Georgia, United States, best predicted by models including both per capita income and $\mathrm{PM}_{10}$ for females, but only per capita income for males suggesting a potential role of $\mathrm{PM}_{10}$ in the etiology of MS in females [15].

While etiologic inferences cannot be drawn from our analyses, the observed pattern may promote the hypothesis of a direct or indirect link between air pollution and MS. In Tehran, air pollution levels are high enough to have a visible impact on air, reducing also the sun light intensity. Thus, one may hypothesize a link between reduced sun light exposure due to air pollution and MS. Vitamin D synthesis under the influence of UVB (Ultraviolet-B) in the skin is the major source for the body requirements, while dietary sources of vitamin $\mathrm{D}$ are responsible for a small portion of the requisites. Interestingly, a previous correlation study reported lower mean serum $25(\mathrm{OH})$ vitamin $\mathrm{D}$ concentrations in a population living in more polluted areas as compared to those living in the less polluted area [11]. Hosseinpanah et al. [12]

Neuroepidemiology 2014;43:233-238 

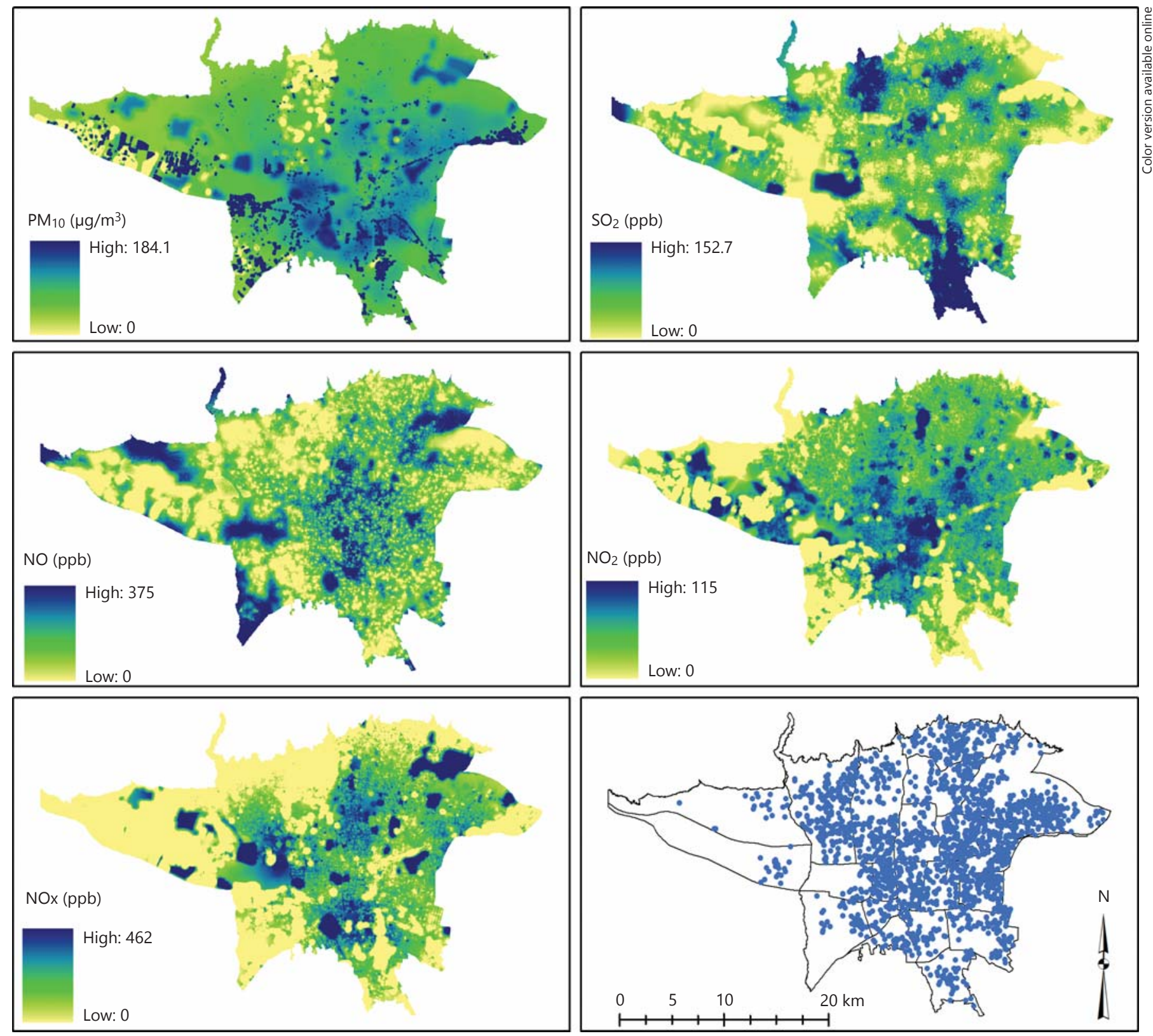

Fig. 1. Particulate matter $\leq 10 \mu \mathrm{m}\left(\mathrm{PM}_{10}\right)$, sulfur dioxide $\left(\mathrm{SO}_{2}\right)$, nitrogen oxide $(\mathrm{NO})$, nitrogen dioxide $\left(\mathrm{NO}_{2}\right)$, and nitrogen oxides (NOx) levels estimated by land use regression models. The last figure shows on the Tehran map locations where there is a high prevalence of MS.

found that the ground level of UVB was significantly higher in the low polluted area (Ghazvin) as compared with Tehran. They also reported that the average blood vitamin D levels were significantly higher in women from the low polluted area and was related to the degree of haze or pollution over these cities [12]. As both studies were merely comparisons between low and high pollution levels, the observed correlations may be explained by other factors that differ between areas with low and high pollution levels. However, a study in Isfahan, a highly prevalent region for MS in Iran [28], revealed significant negative associations between ultraviolet radiation (UVB) and air quality index (AQI). In essence, UVB was positively associated with $25(\mathrm{OH})$ vitamin $\mathrm{D}$ levels in mothers and their neonates in Isfahan, and the AQI had an inverse and independent association [29]. 
Our data did not facilitate the direct investigation of the association between MS relapses and short-term exposure to air pollution, which is another possible hypothesis of relevance in the course of this chronic disease. Oikonen et al. found MS relapses to be in the highest quartile following peak levels of $\mathrm{PM}_{10}, \mathrm{CO}, \mathrm{NOx}$ and $\mathrm{SO}_{2}$ in south-western Finland, after excluding the acidic gases $\left(\mathrm{NOx}\right.$ and $\left.\mathrm{SO}_{2}\right)$ and due to their strong interaction with other environmental variables, MS relapses were only associated with peak amounts in $\mathrm{PM}_{10}$ level [16]. In a further study, Oikonen et al. [17] showed relapses to be more frequent following high levels of $\mathrm{PM}_{10}$ in MS patients not using $\beta$-interferon. MS relapses occurred over twice as often following high numbers of adenovirus infections in this group, while in patients receiving $\beta$-interferon, therapy protected against an enhanced susceptibility to infections caused by $\mathrm{PM}_{10}$ [17]. Hence, inhalable particles could contribute to seasonal variation in MS relapse occurrence possibly by predisposing to airway infections. Prolonged exposure to moderate levels of diesel engine exhaust, a major contributor to particulate air pollution [30], induced a neuroinflammatory response in different regions of the rat brain, TNF- $\alpha$ and IL-1 $\alpha$ protein levels and were specifically increased in the striatum of rat brains [31].

The design of this study limits the ability to draw any etiologic conclusions. Although we have individual-level data available for MS patients, the cases are not derived from a prospective cohort nor is the general population thus the denominator - defined. Therefore, at this stage, we cannot derive spatially resolved incidence or prevalence rates. The observed correlation between the spatial clustering of MS cases and the spatial patterns of major air pollutants may be confounded by a range of other spatially correlated variables, which were unavailable in this study, such as other environmental factors or factors related to population age structure, occupational exposures, or lifestyle co-determined by socio-economic conditions. As recently shown, the socio-economic geography of Tehran explains the substantial discrepancies in life expectancy [32]. It will only be through a prospective population-based cohort with extended follow up that the potential role of air pollutants and its interrelation with the vitamin D pathway in MS pathogenesis may be elucidated. The study is also limited by the fact that both used cluster analysis methods assume that the population density is homogenous in the area.

\section{Conclusions}

This study shows the need to invest in research about the role of ambient air pollutants in the new onset as well as the development of MS. The potential role of long-term exposure to hazardous environmental factors in the MS pathogenesis is not well established but may be relevant in particular in countries with continued environmental degradation and high incidence of MS.

\section{Acknowledgments and Funding}

The authors would like to thank Prof. Nino Künzli (Department of Epidemiology and Public Health, Swiss Tropical and Public Health Institute, Basel, Switzerland) for providing their comments in a previous version of this manuscript. We also greatly admire the efforts of the staff at the IMSS and the GIS bureau of Tehran's post office. The Institute for Environmental Research (IER), an associated institute of Tehran University of Medical Sciences, has supported this study financially and so we acknowledge this gesture.

\section{References}

1 Dutta R, Trapp BD: Pathogenesis of axonal and neuronal damage in multiple sclerosis. Neurology 2007;68(22 suppl 3):S22-S31; discussion S43-S54.

-2 Koch-Henriksen N, Sorensen PS: The changing demographic pattern of multiple sclerosis epidemiology. Lancet Neurol 2010;9:520532.

-3 Heydarpour P, et al: Multiple sclerosis in Tehran, Iran: a joinpoint trend analysis. Mult Scler 2014;20:512.

4 Elhami SR, et al: A 20-year incidence trend (1989-2008) and point prevalence (March 20, 2009) of multiple sclerosis in Tehran, Iran: a population-based study. Neuroepidemiology 2011;36:141-147.
5 Sahraian MA, et al: Multiple sclerosis in Iran: a demographic study of 8,000 patients and changes over time. Eur Neurol 2010;64:331-336.

-6 Lozano R, et al: Global and regional mortality from 235 causes of death for 20 age groups in 1990 and 2010: a systematic analysis for the Global Burden of Disease Study 2010. Lancet 2012;380:2095-2128.

7 Murray CJ, et al: Disability-adjusted life years (DALYs) for 291 diseases and injuries in 21 regions, 1990-2010: a systematic analysis for the Global Burden of Disease Study 2010. Lancet 2012;380:2197-2223.

8 Institute for Health Metrics and Evaluation (IHME): GBD Arrow Diagram. Seattle, WA, IHME, University of Washington, 2013.
Available from http://vizhub.healthdata.org/ irank/arrow.php (accessed July 20, 2014).

$\checkmark 9$ Guimond C, et al: Multiple sclerosis in the Iranian immigrant population of $\mathrm{BC}$, Canada: prevalence and risk factors. Mult Scler 2014; 20:1182-1188.

10 Ebers G: Passive smoking and replication. Eur J Neurol 2008;15:1263-1264.

11 Agarwal KS, et al: The impact of atmospheric pollution on vitamin $\mathrm{D}$ status of infants and toddlers in Delhi, India. Arch Dis Child 2002; 87:111-113.

12 Hosseinpanah F, et al: The effects of air pollution on vitamin D status in healthy women: a cross sectional study. BMC Public Health 2010;10:519. 
13 Amini $\mathrm{H}$, et al: Land use regression models to estimate the annual and seasonal spatial variability of sulfur dioxide and particulate matter in Tehran, Iran. Sci Total Environ 2014;488489:343-353.

14 Hosseinpoor AR, et al: Air pollution and hospitalization due to angina pectoris in Tehran, Iran: a time-series study. Environ Res 2005; 99:126-131.

15 Gregory AC 2nd, et al: Multiple Sclerosis disease distribution and potential impact of environmental air pollutants in Georgia. Sci Total Environ 2008;396:42-51.

16 Oikonen M, et al: Ambient air quality and occurrence of multiple sclerosis relapse. Neuroepidemiology 2003;22:95-99.

17 Oikonen MK, Erälinna JP: Beta-interferon protects multiple sclerosis patients against enhanced susceptibility to infections caused by poor air quality. Neuroepidemiology 2008;30 13-19.

18 Amini $\mathrm{H}$, et al: Correlation of air pollutants with land use and traffic measures in Tehran, Iran: a preliminary statistical analysis for land use regression modeling. J Adv Environ Health Res 2013;1:1-8.
19 Gesler W: The uses of spatial analysis in medical geography: a review. Soc Sci Med 1986;23: 963-973.

20 Gatrell AC, et al: Spatial point pattern analysis and its application in geographical epidemiology. Transactions of the Institute of British Geographers, 1996, pp 256-274.

21 Amini H, et al: Spatial epidemiology and pattern analysis of childhood cancers in Tehran, Iran. Journal of Advances in Environmental Health Research 2014;2:30-37.

22 Johnson J, Kirsch S: Spatial analysis of childhood cancer incidence and electric power line location in Memphis and Shelby County, Tennessee. Southeastern Geographer 1992 32:148-162.

23 Jacquez GM, et al: Global, local and focused geographic clustering for case-control data with residential histories. Environ Health 2005;4:4.

24 Thomas RW: An introduction to quadrat analysis. Geo Abstracts, Norwich, UK, 1977.

25 Amini $\mathrm{H}$, et al: Application of land use regression model to specify the spatial discrepancies of NO2 in Tehran, Iran. Epidemiology 2012; 23(5S).

26 Wang R, et al: Temporal stability of land use regression models for traffic-related air pollution. Atmospheric Environment 2013;64: 312-319.
27 Saei M, Holakouie-Naieni K, Mostafavi E, Sahraian MA, Mahmoodi M, Mansournia MA, Hosseini A: Spatial Analysis of Multiple Sclerosis Disease in Tehran Metro-politan Zone, Iran, 2001-2012. Iranian J Publ Health 2014;43:621-629.

28 Etemadifar M, Maghzi AH: Sharp increase in the incidence and prevalence of multiple sclerosis in Isfahan, Iran. Mult Scler 2011;17: 1022-1027.

29 Kelishadi R, et al: Determinants of hypovitaminosis $\mathrm{d}$ in pregnant women and their newborns in a sunny region. Int J Endocrinol 2013;2013:460970.

30 Donaldson $\mathrm{K}$, et al: Combustion-derived nanoparticles: a review of their toxicology following inhalation exposure. Part Fibre Toxicol 2005;2:10.

31 Gerlofs-Nijland ME, et al: Effect of prolonged exposure to diesel engine exhaust on proinflammatory markers in different regions of the rat brain. Part Fibre Toxicol 2010;7:12.

32 Mokhayeri Y, et al: How within-city socioeconomic disparities affect life expectancy? Results of Urban HEART in Tehran, Iran. Med J Islam Repub Iran 2014;28:80. 